\title{
Application of Computer Vision to Crack Detection of Concrete Structure
}

\author{
Tung-Ching Su
}

\begin{abstract}
Most important civil infrastructures are made of concrete, so accurate information by routine inspection is necessary for structure maintenance. Sometimes temporarily erected scaffoldings are needed for infrastructure inspections. Bridge inspection for example, the inspectors must stand on the platform to examine the underside of a bridge, but such a procedure is risky. At present, several inspection systems coupled with Charge Coupled Devices (CCD) cameras have been developed and applied to infrastructure inspections in order to reduce the danger of accidents to the human inspectors. This paper proposes a computer vision technique based on CCD images to attempt to automatically detect cracks in concrete structure. The experimental result indicates that the optimal accuracies of $90 \%$ and $84 \%$ could be achieved for the training and testing samples, respectively.
\end{abstract}

Index Terms-Crack detection, computer vision, concrete structure, infrastructure inspection.

\section{INTRODUCTION}

Acquisition of accurate information for concrete structure conditions by traditional inspection approaches is usually time-consuming and dangerous because the amount of inspection images is enormous and human inspection is necessary. Due to the extensive developments of computer and image processing techniques, automated inspection is expected to replace human inspection for detecting cracks in concrete structures. Detecting cracks in concrete structures can reveal the major structural problems, such as superficial damage of monolithic construction. The crack detection filters of different sizes had been designed to identify the cracking regions from the inspection images [1]. Principal Component Analysis (PCA)-based algorithm coupled with linear structure modeling was proposed to detect cracks with linear structure in concrete bridge decks with the best detection accuracy of $73 \%$ [2]. For detecting concrete cracks in tunnel, a mobile robot system was built to acquire image data with a Charged Couple Device (CCD) camera [3]. Nevertheless, the mobile robot system was only validated in indoor experimental settings, so further research is encouraged.

Edge-detection algorithms, as well as transform and statistical-based methods, were applied to damage assessment for concrete structure [4]. The texture analysis approach, which provided an overall accuracy of $83.3 \%$, was

Manuscript received February 5, 2013; revised May 7, 2013. This work was supported by the Taiwan National Science Council under Grant 101-2221-E-507-004-.

T. C. Su is with the Department of Civil Engineering and Engineering Management, National Quemoy University, Kinmen, 89250 Taiwan (e-mail: spcyj@nqu.edu.tw).

found to be the most efficient technique for extracting damage information from acoustic imagery. The other statistical texture analyses also had been adopted to extract the texture features, including mean, homogeneity, and dissimilarity, from the grey level co-occurrence matrix in order to detect cracks in concrete with an overall accuracy between 68.7 and 76.5\% [5]. However, such accuracy is unsatisfied for practice applications. In this paper, therefore, a novel computer vision method is presented to attempt to derive an accuracy of crack detection of $80 \%$ at least.

\section{EXPERIMENTAL MATERIALS}

The cracks in the concrete structure were imaged using a common digital camera, which specifications involve $3.15 \times 10^{6}(1536 \times 2048)$ pixels and adjustable focal lengths of $35 \mathrm{~mm}$ through $105 \mathrm{~mm}$. In order to reduce the computation time effectively, each inspection image of $1536 \times 2048$ pixels was resampled to $335 \times 413$ pixels. Fig. 1 shows the sample images of crack and non-crack concrete paving. In this research, 100 images for the concrete paving inspection were adopted as the experimental materials. A half of the 100 images have the patterns of crack and the others show the non-crack patterns. In order to demonstrate the performance of the proposed computer vision method, 50 of the 100 images were selected as the training samples, and the others were the testing samples. Crack and non-crack both were given 25 images in both training and testing samples.

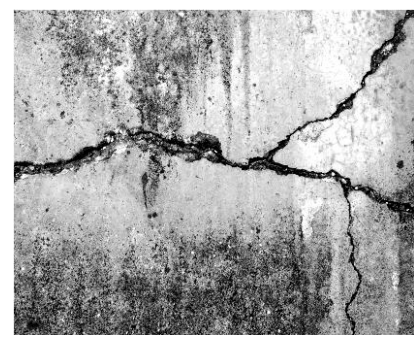

(a) crack

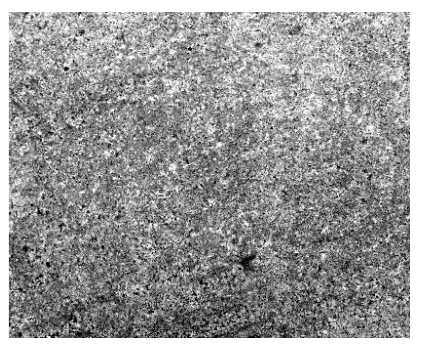

(b) non-crack

Fig. 1. Sample images of the concrete paving inspection.

\section{Methodology}

Fig. 2 is the scheme of the proposed computer vision method for crack detection on concrete structure. Initially, all of the acquired inspection images of concrete structures are transformed into the gray level ones. In other words, color information is not taken into consideration of this research because the cracks are almost darker than their neighborhoods. Su et al. (2011) used opening top-hat operation to attempt to identify the representative morphologies for the multiple kinds of sewer pipe defects [6]. They found that it is extremely difficult to segment the 
representative morphologies of the sewer pipe defects from the inspection images due to the noisy environments. To effectively reduce the interferences of the noisy environments, this research designed the weighted median filters of different sizes to smooth the details of the noisy environments as well as remain the textures of the cracks.

An image opening is applied to the filtered images to enhance the textures of the cracks. Considering the characteristic of irregular line-like structures of cracks, a structure element of line shape is given for the image opening, which is executed in the directions of $0^{\circ}, 30^{\circ}, 60^{\circ}, 90^{\circ}, 120^{\circ}$, and $150^{\circ}$ for each gray level image. Overlapping the opening operated images of the different directions into a single one is to be transformed into a binary image by Otsu's thresholding.

After the binary transformation (or called image segmentation), a large segmented region has high probability of being considered as a crack compared to a segmented noisy with a relatively small region. In addition, the eccentricity values of 0 and 1 represent the shapes of circle and line, respectively. A segmented region with an eccentricity approximating to 1 means high probability of being considered as a crack. Therefore, two morphological features, including area and eccentricity, are measured for each segmented region. Based on the measured morphological features, several criteria will be established and used to determine either crack or non-crack pattern in image.

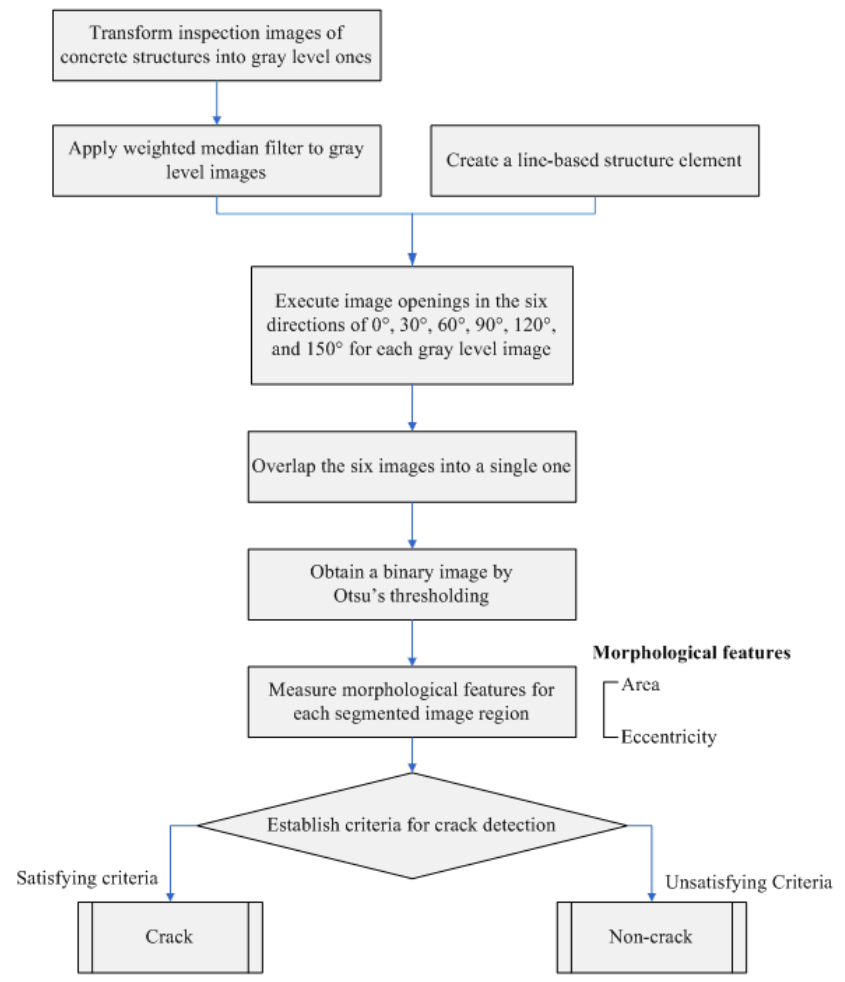

Fig. 2. Scheme of the proposed computer vision method for crack detection on concrete structure.

\section{A. Weighted Median Filter}

Median filter is one of the smoothing filters to remove environmental noise from an image. Unfortunately, textures of interested image regions would be removed simultaneously while median filter is operated so to cause blurred textures [7]. To tackle the above problem, weighted median filter was presented [8], [9]. The principle of weighted median filter is similar to that of median filter but the pixel value at the central position of weighted median filter is duplicated $k$ times. Thus, weighted median filter can effectively remove environmental noise and remain texture as completely as possible [10].

\section{B. Image Opening}

Image opening is one of the mathematical morphology operations to detect interested image region. Before an operation of image opening, the type of structural element needs to be determined. The determination of the type of structural element depends upon the kind of interested shape. In other words, the structural element is used to assist the operation of image opening in enhancing the image regions similar to the structural element. Due to the morphological characteristic of irregular line-like of crack, this research introduces a linear structural element of 15 pixels into the operation of image opening in the six directions, including $0^{\circ}$, $30^{\circ}, 60^{\circ}, 90^{\circ}, 120^{\circ}$, and $150^{\circ}$.

\section{Otsu's Thresholding}

Otsu's thresholding, which is a thresholding method based discriminant analysis, determines the optimal thresholds for image opening by maximizing the following measure of class separability [11]:

$$
D(T)=\frac{P_{1}(T) P_{2}(T)\left[m_{1}(T)-m_{2}(T)\right]^{2}}{P_{1}(T) \sigma_{1}^{2}(T)+P_{2}(T) \sigma_{2}^{2}(T)}
$$

The parameters in Eq. (1) were described in the literature of Yang and $\mathrm{Su}$ [12]. By maximizing the criterion function in Eq. (1), the means of the light and dark image regions can be separated as well as possible and the variances of the two image regions can be minimized. In this paper, Otsu's thresholding is adopted to transform the opening operated images into the binary ones to segment cracks.

\section{Measurement of Morphological Features}

In this paper, the measured morphology of the segmented regions includes area and eccentricity. If a segmented region consisting of $m$ pixels, its area is simply recorded as $m$. Eccentricity means the ratio of foci to major axis length and is written as

$$
e=f / L
$$

where $e$ means eccentricity, $f$ and $L$ represents foci and major axis length, respectively.

\section{E. Establishment of Criteria for Crack Detection}

Based on the measured morphological features, several criteria were established for crack detection in which a segmented region satisfying one of the criteria is regarded as portion of crack. In addition, giving different weights $k$ for the weighted median filtering would cause different image segmentation results so to increase the uncertainty of establishing criteria for crack detection. In this paper, establishment of criteria for crack detection is also a solution for an uncertainty and optimization problem.

Sensitivity analysis has been widely applied to solve uncertainty or optimization problems [13], [14]. In several literatures, sensitivity analysis was also presented to facilitate the crack detections of structures. Quek et al. (2001) 
examined the sensitivity of the wavelet techniques, including Gabor and Haar wavelets, in the detection of cracks in beam structures [15]. The experimental result indicates that Haar wavelets exhibit superior performance for detection of discrete cracks. Naseralavi et al. (2011) also proposed a method based on sensitivity analysis of the structure for structural damage detection using natural frequencies [16]. Based on the above literatures, sensitivity analysis has been demonstrated to be able to effectively offer optimal parameters for decision problems. There are $i$ criteria and $j$ weights to be determined so to have $i * j$ times of accuracy computation remaining for solving such problem.

\section{EXPERIMENTAL RESULTS}

In order to determine the optimal parameters, including weight of weighted median filter, area, and eccentricity, for crack detection, a preliminary survey to the derived morphological features is necessary. In the preliminary survey, the weight $k$ was given as one, i.e. equivalent weight, and applied to the two images in Fig. 1. The segmentation results by the steps of $A$ through $C$ in section III are shown as Fig. 3. Compared with the two pairs of the segmentation, the larger weighted median filter significantly reduces the number of the segmented regions of noisy environment. Table I is a statistic list for the number of the segmented regions in Fig. 3. Table I is seen that the segmented regions, which consist of less than 50 pixels or have the eccentricities of less than 0.8 , are the majority for each binary image. Unfortunately, most of those segmented regions belong to noisy environment. In other words, a segmented region satisfying the criterion, i.e. area threshold $\geqq 50$ pixels coupled with eccentricity threshold $\geqq 0.8$, has a higher probability to be portion of crack. Based on the above result, a sensitivity analysis was schemed for finding out the appropriate criteria for crack detection.

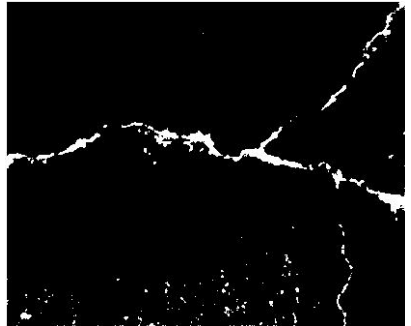

(a1)

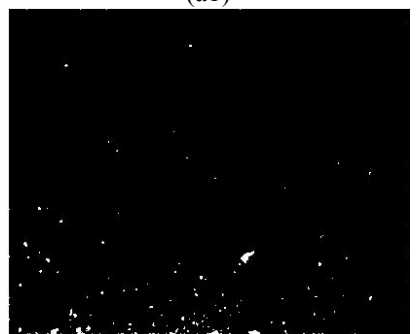

(b1)

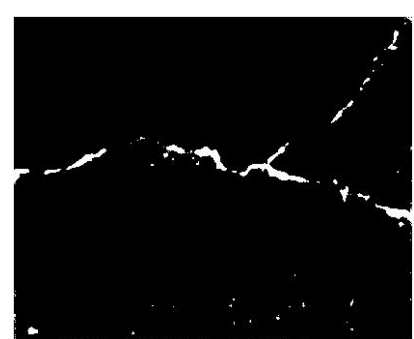

(a2)

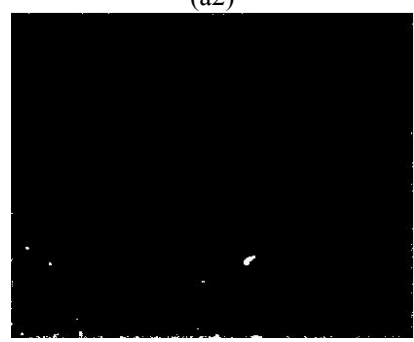

(b2)
Fig. 3. Segmentation results of the sample images in Fig. 1 using $3 \times 3$ weighted median filters (see (a1) and (b1)) and $5 \times 5$ weighted median filters (see (a2) and (b2)).

\section{A. Training of Crack Detection}

Tables II shows a sensitivity analysis for the 50 training samples using the weighted median filters with $k=1$. According to each criterion listed in the tables, the accuracies of crack and non-crack detections were estimated. Under an area threshold, the higher the given eccentricity threshold is, the lower the accuracy of crack detection is obtained. On the contrary, the derived accuracy of non-crack detection is ascent once the higher eccentricity threshold is given. Thus, the crack and non-crack detections have a trade-off relationship.

In Table II through Table VI, the weighted median filters with the different weights $k$, including $1,3,5,7$, and 9, were tested. The sensitivity analyses demonstrate that the weight $k$ for the weighted median filter has slight effect on crack detection based on the insignificant difference among the best overall accuracies of 0.80 through 0.90 . Moreover, six criteria consisting of area (in pixel) threshold and eccentricity threshold, including $(\geqq 50, \geqq 0.95),(\geqq 100, \geqq 0.90),(\geqq 100$, $\geqq 0.95),(\geqq 200, \geqq 0.80),(\geqq 200, \geqq 0.85)$, and ( $\geqq 200, \geqq$ $0.90)$, can lead to the best overall accuracies under the condition of various weights setting in filtering.

TABLE I: NUMBER OF THE SEGMENTED REGIONS IN FIG. 3

\begin{tabular}{lcccc}
\hline \hline & \multicolumn{4}{c}{ Figure ID } \\
Area (pixels) & $(\mathrm{a} 1)$ & $(\mathrm{a} 2)$ & $(\mathrm{b} 1)$ & $(\mathrm{b} 2)$ \\
\hline$\geqq 200$ & 5 & 4 & 0 & 0 \\
$200>\sim \geqq 150$ & 1 & 0 & 0 & 0 \\
$150>\sim \geqq 100$ & 3 & 2 & 0 & 0 \\
$100>\sim \geqq 50$ & 4 & 5 & 1 & 1 \\
$<50$ & 166 & 106 & 139 & 85 \\
Eccentricity & & & & \\
\hline $1.0 \geqq \sim \geqq 0.95$ & 10 & 5 & 1 & 4 \\
$0.95>\sim \geqq 0.90$ & 10 & 13 & 7 & 7 \\
$0.90>\sim \geqq 0.85$ & 41 & 32 & 37 & 20 \\
$0.85>\sim \geqq 0.80$ & 6 & 2 & 3 & 1 \\
$<0.80$ & 112 & 65 & 92 & 54 \\
\hline \hline
\end{tabular}

TABLE II: SENSITIVITY ANALYSIS FOR THE 50 TRAINING SAMPLES USING A $5 \times 5$ WEIGHTED MEDIAN FILTER WITH $K=1$

\begin{tabular}{|c|c|c|c|c|}
\hline \multicolumn{2}{|c|}{ Criterion } & \multirow{2}{*}{$\begin{array}{c}\text { Accuracy of } \\
\text { crack } \\
\text { detection }\end{array}$} & \multirow{2}{*}{$\begin{array}{c}\text { Accuracy of } \\
\text { Non-crack } \\
\text { detection }\end{array}$} & \multirow{2}{*}{$\begin{array}{l}\text { Overall } \\
\text { accuracy }\end{array}$} \\
\hline $\begin{array}{c}\text { Area } \\
\text { (pixels) }\end{array}$ & Eccentricity & & & \\
\hline \multirow[t]{4}{*}{$>=50$} & $>=0.80$ & 1.00 & 0.48 & 0.74 \\
\hline & $>=0.85$ & 1.00 & 0.52 & 0.76 \\
\hline & $>=0.90$ & 1.00 & 0.64 & 0.82 \\
\hline & $>=0.95$ & 0.96 & 0.76 & 0.86 \\
\hline \multirow[t]{4}{*}{$>=100$} & $>=0.80$ & 0.92 & 0.84 & 0.88 \\
\hline & $>=0.85$ & 0.92 & 0.84 & 0.88 \\
\hline & $>=0.90$ & 0.92 & 0.88 & 0.90 \\
\hline & $>=0.95$ & 0.84 & 0.88 & 0.86 \\
\hline \multirow[t]{4}{*}{$>=150$} & $>=0.80$ & 0.84 & 0.84 & 0.84 \\
\hline & $>=0.85$ & 0.84 & 0.84 & 0.84 \\
\hline & $>=0.90$ & 0.84 & 0.88 & 0.86 \\
\hline & $>=0.95$ & 0.72 & 0.88 & 0.80 \\
\hline \multirow[t]{4}{*}{$>=200$} & $>=0.80$ & 0.76 & 0.88 & 0.82 \\
\hline & $>=0.85$ & 0.76 & 0.88 & 0.82 \\
\hline & $>=0.90$ & 0.76 & 0.92 & 0.84 \\
\hline & $>=0.95$ & 0.56 & 0.92 & 0.74 \\
\hline
\end{tabular}


TABLE III: SENSITIVITY ANALYSIS FOR THE 50 TRAINING SAMPLES USING A $5 \times 5$ WeIGHTED MEdian FILTER WITH $K=3$

\begin{tabular}{|c|c|c|c|c|}
\hline \multicolumn{2}{|c|}{ Criterion } & \multirow{2}{*}{$\begin{array}{l}\text { Accuracy of } \\
\text { crack } \\
\text { detection }\end{array}$} & \multirow{2}{*}{$\begin{array}{c}\text { Accuracy of } \\
\text { non-crack } \\
\text { detection }\end{array}$} & \multirow{2}{*}{$\begin{array}{c}\text { Overall } \\
\text { accuracy }\end{array}$} \\
\hline $\begin{array}{c}\text { Area } \\
\text { (pixels) }\end{array}$ & Eccentricity & & & \\
\hline \multirow[t]{4}{*}{$>=50$} & $>=0.80$ & 0.96 & 0.52 & 0.74 \\
\hline & $>=0.85$ & 0.96 & 0.52 & 0.74 \\
\hline & $>=0.90$ & 0.96 & 0.60 & 0.78 \\
\hline & $>=0.95$ & 0.92 & 0.76 & 0.84 \\
\hline \multirow[t]{4}{*}{$>=100$} & $>=0.80$ & 0.92 & 0.72 & 0.82 \\
\hline & $>=0.85$ & 0.92 & 0.76 & 0.84 \\
\hline & $>=0.90$ & 0.92 & 0.80 & 0.86 \\
\hline & $>=0.95$ & 0.88 & 0.88 & 0.88 \\
\hline \multirow[t]{4}{*}{$>=150$} & $>=0.80$ & 0.84 & 0.80 & 0.82 \\
\hline & $>=0.85$ & 0.84 & 0.80 & 0.82 \\
\hline & $>=0.90$ & 0.84 & 0.84 & 0.84 \\
\hline & $>=0.95$ & 0.76 & 0.88 & 0.82 \\
\hline \multirow[t]{4}{*}{$>=200$} & $>=0.80$ & 0.80 & 0.84 & 0.82 \\
\hline & $>=0.85$ & 0.80 & 0.84 & 0.82 \\
\hline & $>=0.90$ & 0.80 & 0.88 & 0.84 \\
\hline & $>=0.95$ & 0.64 & 0.92 & 0.78 \\
\hline
\end{tabular}

TABLE IV: SENSITIVITY ANALYSIS FOR THE 50 TRAINING SAMPLES USING A $5 \times 5$ WeIGHTED MEdian FILTER WITH $K=5$

\begin{tabular}{|c|c|c|c|c|}
\hline \multicolumn{2}{|c|}{ Criterion } & \multirow{2}{*}{$\begin{array}{c}\text { Accuracy of } \\
\text { crack } \\
\text { detection }\end{array}$} & \multirow{2}{*}{$\begin{array}{c}\text { Accuracy of } \\
\text { non-crack } \\
\text { detection }\end{array}$} & \multirow{2}{*}{$\begin{array}{c}\text { Overall } \\
\text { accuracy }\end{array}$} \\
\hline $\begin{array}{c}\text { Area } \\
\text { (pixels) }\end{array}$ & Eccentricity & & & \\
\hline \multirow{4}{*}{$>=50$} & $>=0.80$ & 1.00 & 0.40 & 0.70 \\
\hline & $>=0.85$ & 1.00 & 0.44 & 0.72 \\
\hline & $>=0.90$ & 1.00 & 0.48 & 0.74 \\
\hline & $>=0.95$ & 0.96 & 0.76 & 0.86 \\
\hline \multirow{4}{*}{$>=100$} & $>=0.80$ & 0.96 & 0.64 & 0.80 \\
\hline & $>=0.85$ & 0.96 & 0.68 & 0.82 \\
\hline & $>=0.90$ & 0.92 & 0.72 & 0.82 \\
\hline & $>=0.95$ & 0.76 & 0.88 & 0.82 \\
\hline \multirow{4}{*}{$>=150$} & $>=0.80$ & 0.88 & 0.80 & 0.84 \\
\hline & $>=0.85$ & 0.88 & 0.80 & 0.84 \\
\hline & $>=0.90$ & 0.84 & 0.84 & 0.84 \\
\hline & $>=0.95$ & 0.72 & 0.88 & 0.80 \\
\hline \multirow{4}{*}{$>=200$} & $>=0.80$ & 0.88 & 0.84 & 0.86 \\
\hline & $>=0.85$ & 0.88 & 0.84 & 0.86 \\
\hline & $>=0.90$ & 0.80 & 0.88 & 0.84 \\
\hline & $>=0.95$ & 0.60 & 0.92 & 0.76 \\
\hline
\end{tabular}

\section{B. Testing of Crack Detection}

Based on the above six criteria, the accuracies of the crack and non-crack detections were also estimated for the 50 testing samples. According to the found optimal weight, area, and eccentricity, the testing result of crack and non-crack detections in Table VII show that the accuracies of crack and non-crack detections both range from 0.72 to 0.88 . Area thresholds $\geqq 50$ or 100 pixels coupled with eccentricity threshold $\geqq 0.95$ could lead to the better overall accuracies above 0.80 .

Comparing the training with testing samples, the largest difference of the overall accuracies is $12 \%$ while $k=1$, and area threshold $\geqq 100$ pixels coupled with eccentricity threshold $\geqq 0.90$ are applied. The difference of $12 \%$ indicates that the testing samples compared to the training ones have six omitted samples (three for crack detection and three for non-crack detection). On the contrary, the least difference of the overall accuracies between the training and testing samples is $2 \%$ while $k=9$, and area threshold $\geqq 200$ pixels coupled with eccentricity threshold $\geqq 0.90$ are adopted. The overall accuracy difference of $2 \%$ is caused by that the testing samples compared to the training samples have three more omitted samples for crack detection but two less omitted samples for non-crack detection. Consequently, this paper demonstrates that the different criteria for crack detection could result in slight omission errors that cause no great effect on crack defection.

TABLE V: Sensitivity ANALYSiS For the 50 TRAining SAMPLES USING A $5 \times 5$ WEIGHTED MEDIAN FILTER WITH $K=7$

\begin{tabular}{|c|c|c|c|c|}
\hline \multicolumn{2}{|c|}{ Criterion } & \multirow{2}{*}{$\begin{array}{c}\text { Accuracy of } \\
\text { crack } \\
\text { detection }\end{array}$} & \multirow{2}{*}{$\begin{array}{c}\text { Accuracy of } \\
\text { non-crack } \\
\text { detection }\end{array}$} & \multirow{2}{*}{$\begin{array}{c}\text { Overall } \\
\text { accuracy }\end{array}$} \\
\hline $\begin{array}{c}\text { Area } \\
\text { (pixels) }\end{array}$ & Eccentricity & & & \\
\hline \multirow{4}{*}{$>=50$} & $>=0.80$ & 1.00 & 0.36 & 0.68 \\
\hline & $>=0.85$ & 1.00 & 0.36 & 0.68 \\
\hline & $>=0.90$ & 1.00 & 0.40 & 0.70 \\
\hline & $>=0.95$ & 1.00 & 0.64 & 0.82 \\
\hline \multirow{4}{*}{$>=100$} & $>=0.80$ & 0.96 & 0.52 & 0.74 \\
\hline & $>=0.85$ & 0.96 & 0.56 & 0.76 \\
\hline & $>=0.90$ & 0.92 & 0.64 & 0.78 \\
\hline & $>=0.95$ & 0.84 & 0.76 & 0.80 \\
\hline \multirow{4}{*}{$>=150$} & $>=0.80$ & 0.92 & 0.72 & 0.82 \\
\hline & $>=0.85$ & 0.92 & 0.72 & 0.82 \\
\hline & $>=0.90$ & 0.84 & 0.84 & 0.84 \\
\hline & $>=0.95$ & 0.80 & 0.84 & 0.82 \\
\hline \multirow{4}{*}{$>=200$} & $>=0.80$ & 0.92 & 0.80 & 0.86 \\
\hline & $>=0.85$ & 0.92 & 0.80 & 0.86 \\
\hline & $>=0.90$ & 0.80 & 0.88 & 0.84 \\
\hline & $>=0.95$ & 0.64 & 0.88 & 0.76 \\
\hline
\end{tabular}

TABLE VI: SENSITIVITY ANALYSIS FOR THE 50 TRAINING SAMPLES USING A $5 \times 5$ WEIGHTED MEDIAN FILTER WITH $K=9$

\begin{tabular}{|c|c|c|c|c|}
\hline \multicolumn{2}{|c|}{ Criterion } & \multirow{2}{*}{$\begin{array}{c}\text { Accuracy of } \\
\text { crack } \\
\text { detection }\end{array}$} & \multirow{2}{*}{$\begin{array}{c}\text { Accuracy of } \\
\text { non-crack } \\
\text { detection }\end{array}$} & \multirow{2}{*}{$\begin{array}{l}\text { Overall } \\
\text { accuracy }\end{array}$} \\
\hline $\begin{array}{c}\text { Area } \\
\text { (pixels) }\end{array}$ & Eccentricity & & & \\
\hline \multirow{4}{*}{$>=50$} & $>=0.80$ & 1.00 & 0.28 & 0.64 \\
\hline & $>=0.85$ & 1.00 & 0.28 & 0.64 \\
\hline & $>=0.90$ & 1.00 & 0.32 & 0.66 \\
\hline & $>=0.95$ & 1.00 & 0.60 & 0.80 \\
\hline \multirow{4}{*}{$>=100$} & $>=0.80$ & 0.96 & 0.44 & 0.70 \\
\hline & $>=0.85$ & 0.96 & 0.44 & 0.70 \\
\hline & $>=0.90$ & 0.92 & 0.44 & 0.68 \\
\hline & $>=0.95$ & 0.84 & 0.68 & 0.76 \\
\hline \multirow{4}{*}{$>=150$} & $>=0.80$ & 0.96 & 0.48 & 0.72 \\
\hline & $>=0.85$ & 0.96 & 0.48 & 0.72 \\
\hline & $>=0.90$ & 0.92 & 0.56 & 0.74 \\
\hline & $>=0.95$ & 0.80 & 0.68 & 0.74 \\
\hline \multirow{4}{*}{$>=200$} & $>=0.80$ & 0.96 & 0.64 & 0.80 \\
\hline & $>=0.85$ & 0.92 & 0.64 & 0.78 \\
\hline & $>=0.90$ & 0.88 & 0.72 & 0.80 \\
\hline & $>=0.95$ & 0.72 & 0.84 & 0.78 \\
\hline
\end{tabular}


TABLE VII: TESTING RESULT OF THE CRACK AND NON-CRACK DETECTIONS FOR THE 50 TESTING SAMPLES

\begin{tabular}{cccccc}
\hline \hline \multirow{2}{*}{$\begin{array}{c}K \\
\text { value }\end{array}$} & \multicolumn{2}{c}{ Criterion } & $\begin{array}{c}\text { Accuracy } \\
\text { of crack } \\
\text { detection }\end{array}$ & $\begin{array}{c}\text { Accuracy } \\
\text { of } \\
\text { non-crack } \\
\text { netection }\end{array}$ & $\begin{array}{c}\text { Overall } \\
\text { accuracy }\end{array}$ \\
\cline { 2 - 3 } & $\begin{array}{c}\text { Area } \\
\text { (pixels }\end{array}$ & Eccentricity & & & \\
\hline 1 & $>=100$ & $>=0.90$ & 0.80 & 0.76 & 0.78 \\
3 & $>=100$ & $>=0.95$ & 0.72 & 0.88 & 0.80 \\
5 & $>=50$ & $>=0.95$ & 0.84 & 0.80 & 0.82 \\
5 & $>=200$ & $>=0.80$ & 0.76 & 0.80 & 0.78 \\
5 & $>=200$ & $>=0.85$ & 0.72 & 0.80 & 0.76 \\
7 & $>=200$ & $>=0.80$ & 0.72 & 0.80 & 0.76 \\
7 & $>=200$ & $>=0.85$ & 0.72 & 0.84 & 0.78 \\
9 & $>=50$ & $>=0.95$ & 0.88 & 0.80 & 0.84 \\
9 & $>=200$ & $>=0.80$ & 0.76 & 0.72 & 0.74 \\
9 & $>=200$ & $>=0.90$ & 0.76 & 0.80 & 0.78 \\
\hline \hline
\end{tabular}

\section{CONCLUSION}

This paper proposed a computer vision method, in which weighted median filter, image opening, Otsu's thresholding, and measurement of morphological features are executed, to automatically detect cracks in concrete structure. There were 50 frames of the concrete paving images to be the training samples, and another 50 frames were imaged to be the testing samples. In this paper, the $5 \times 5$ weighted median filter has the better performance of assisting the computer vision method in crack detection than the $3 \times 3$ one. Moreover, this paper demonstrated that different weights $k$ do not have great impact to crack detection.

The morphological features, including area and eccentricity, were measured for each segmented image region. Based on the measured morphological features, a sensitivity analysis was applied to establish several criteria for crack detection. The analysis result demonstrates that the optimal overall accuracies of $90 \%$ (using $k=1$, and area threshold $\geqq 100$ pixels coupled with eccentricity threshold $\geqq 0.90$ ) and $84 \%$ (using $k=9$, and area threshold $\geqq 50$ pixels coupled with eccentricity threshold $\geqq 0.95$ ) can be obtained for the training and testing samples, respectively. Thus, the proposed computer vision method is effective to automatically detect cracks in concrete structure.

\section{ACKNOWLEDGMENT}

The author Tung-Ching Su appreciates the supporting of a grant (101-2221-E-507-004-) from Studies on Engineering Issues of Kinmen Bridge - Automated Inspection Issues funded by Taiwan National Science Council.

\section{REFERENCES}

[1] S. K. Sinha and P. W. Fieguth, "Segmentation of buried concrete pipe images," Automation in Construction, vol. 15, no. 1, pp. 58-72, 2006.

[2] I. Abdel-Qader, S. Pashaie-Rad, O. Abudayyeh, and S. Yehia, "PCA-Based algorithm for unsupervised bridge crack detection," Advances in Engineering Software, vol. 37, no. 12, pp. 771-778, 2006.

[3] S. N. Yu, J. H. Jang, and C. S. Han, "Auto inspection system using a mobile robot for detecting concrete cracks in a tunnel," Automation in Construction, vol. 16, no. 3, pp. 255-261, 2007.

[4] S. Kabir, P. Rivard, D .C. He, and P. Thivierge, "Damage assessment for concrete structure using image processing techniques on acoustic borehole imagery," Construction and Building Materials, vol. 23, no. 10, pp. 3166-3174, 2009.

[5] S. Kabir, "Imaging-based detection of AAR induced map-crack damage in concrete structure," NDT\&E International, vol. 43, no. 6, pp. 461-469, 2010.

[6] T. C. Su, M. D. Yang, T. C. Wu, and J. Y. Lin, "Morphological segmentation based on edge detection for sewer pipe defects on CCTV images," Expert Systems with Applications, vol. 38, no. 10, pp. 13094-13114, 2011.

[7] F. Y. Shih, Image Processing and Pattern Recognition, Wiley, New Jersey, 2010.

[8] P. D. Wendt, E. J. Coyle, and N. C. Gallagher Jr., "Stack filter," IEEE Trans. Acoust. Speech Signal Process, vol. 34, no. 4, pp. 898-911, 1986.

[9] O. Yli-Harja, J. Astola, and Y. Neuvo, "Analysis of the properties of median and weighted median filters using threshold logic and stack filter representation," IEEE Trans. Acoust. Speech Signal Process, vol. 39, no. 2, pp. 395-410, 1991.

[10] R. Yang, M. Gabbouj, and Y. Neuvo, "Fast algorithms for analyzing and designing weighted median filters," Signal Processing, vol. 41, no. 2, pp. 135-152, 1995.

[11] H. Yan, "Unified formulation of a class of image thresholding techniques," Pattern Recognition, vol. 29, no. 12, pp. 2025-2032, 1996.

[12] M. D. Yang, and T. C. Su, "Segmenting ideal morphologies of sewer pipe defects on CCTV images for automated diagnosis," Expert Systems with Applications, vol. 36, no. 2, pp. 3562-3573, 2009.

[13] Cs. Mészáros, and T. Rapcsák, "On sensitivity analysis for a class of decision systems," Decision Support Systems, vol. 16, no. 3, pp. 231-240, 1996.

[14] J. K. Ravalico, H. R. Maier, and G. C. Dandy, "Sensitivity analysis for decision-making using the MORE method-A Pareto approach," Reliability Engineering \& System Safety, vol. 94, no. 7, pp. 1229-1237, 2009.

[15] S. T. Quek, Q. Wang, L. Zhang, and K. K. Ang, "Sensitivity analysis of crack detection in beams by wavelet technique," International Journal of Mechanical Sciences, vol. 43, no. 12, pp. 2899-2910, 2001.

[16] S. S. Naseralavi, M. J. Fadaee, and J. Salajegheh, "Subset solving algorithm: A novel sensitivity-based method for damage detection of structures," Applied Mathematical Modelling, vol. 35, no. 5, pp. 2232-2252, 2011.

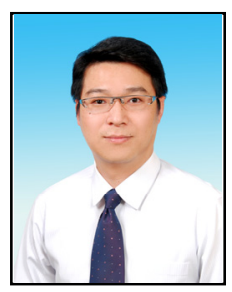

Tung-Ching Su received his M.S. and Ph.D. degrees in civil engineering from National Chung Hsing University (NCHU), Taichung, Taiwan, in 2002 and 2007, respectively. From September 2008 through August 2009, he worked as a post doctor with the Environmental Restoration and Disaster Reduction Research Center at NCHU. Since September 2009, he is an assistance professor of National Quemoy University, Kinmen, Taiwan. His research interests focus on remote sensing, image processing, pattern recognition, and expert systems. 